\title{
INFESTATION HUMAINE PAR TRICHOSTRONGYLUS VITRINUS AU MAROC
}

\author{
J. POIRRIEZ*, E. DEI-CAS*, E. GUEVART**, M. ABDELLATIFI*, P. GIARD** et A. VERNES*
}

RÉSUMÉ. Les auteurs décrivent un cas d'infestation par Trichostrongylus vitrinus chez une marocaine âgée de 53 ans.

\section{Human infection with Trichostrongylus vitrinus in Morocco}

SUMMARY. A case of Trichostrongylus vitrinus infection in a 53-year-old Moroccan woman is reported.

Bien que Becmeur et coll. (1950) aient noté la présence d'œufs de strongles dans les selles de mineurs au Maroc, nous n'avons pu trouver aucune publication confirmant formellement l'existence de cas humains de trichostrongylose dans ce pays. En 1982, Becquet et coll. ont identifié 14 mâles de Trichostrongylus colubriformis et 1 mâle de $T$. axei dans les selles d'un étudiant marocain âgé de 26 ans (originaire de Tétouan), examiné à Lille 6 mois après son arrivée en France ; par conséquent, une contamination locale ne pouvait être totalement exclue, même si ce patient expliquait avoir passé ses vacances au nord du Maroc dans des villages où les agriculteurs utilisent des engrais animaux et humains dans leurs jardins. La présente observation confirme l'existence de la trichostrongylose humaine au Maroc, avec identification d'une $3^{\mathrm{e}}$ espèce.

\section{Observation}

Le 26 octobre 1982, une marocaine âgée de 53 ans (originaire de Meknès) arrive en France pour la première fois. Dix jours plus tard, elle est hospitalisée par suite d'asthénie, d'amaigrissement et de douleurs dans les membres inférieurs. Le bilan biologique sanguin révèle un syndrome inflammatoire et les examens radiologiques

* Service de Parasitologie, Faculté de Médecine, 1 Place de Verdun, F 59045 Lille Cedex, et INSERM, U. 42, Domaine du Certia, 369 Rue Jules-Guesde, F 59650 Villeneuve d'Ascq.

** Service de Médecine Générale, Hôpital de la Charité, 196 Boulevard Montebello, F 59037 Lille Cedex. Accepté le 24 janvier 1984 . 
décèlent une arthrose de la hanche. Les examens parasitologiques de selles montrent la présence de kystes d'Entamoeba histolytica, d'E. coli et d'E. hartmanni, ainsi que des œufs de Trichostrongylus. La malade est traitée durant 7 jours par le métronidazole (Flagyl $\left.{ }^{\circledR}\right)$; le jour suivant, elle reçoit une dose de 5 grammes d'hydroxynaphtoate de béphénium (Alcopar ${ }^{\circledR}$ ) et, 3 heures plus tard, une purgation saline, selon Ghadirian et coll. (1968). L'examen des selles recueillies durant les 24 heures suivantes permet de trouver 3 Trichostrongylus adultes. La bourse caudale et les spicules (fig. 1 et fig. 2) de 2 mâles permettent de les identifier comme T. vitrinus Looss, 1905 (Clapham, 1947 ; Looss, 1905 ; Nagaty, 1932 ; Skrjabin, 1954). Leurs mensurations respectives sont les suivantes : longueur, 3,7 et $3,3 \mathrm{~mm}$; largeur maximale, 0,096 et $0,094 \mathrm{~mm}$; longueur des spicules, $0,156,0,150,0,140$ et $0,135 \mathrm{~mm}$; longueur du gubernaculum 0,075 et $0,072 \mathrm{~mm}$; longueur de la côte dorsale, 0,052 et $0,050 \mathrm{~mm}$ (bifurquée en 2 branches à son dernier quart). Le $3^{\mathrm{e}}$ ver, une femelle, présente les mensurations suivantes : longueur, $3 \mathrm{~mm}$; largeur maximale, 0,093 mm; l'anus est situé à $0,705 \mathrm{~mm}$ de la vulve et à $0,073 \mathrm{~mm}$ de l'extrémité caudale ; la vulve est une fente longitudinale longue de $0,050 \mathrm{~mm}$; les 2 utérus contiennent au total 9 œufs mesurant de 0,093 à 0,123 mm de long (moyenne : 0,105 mm).
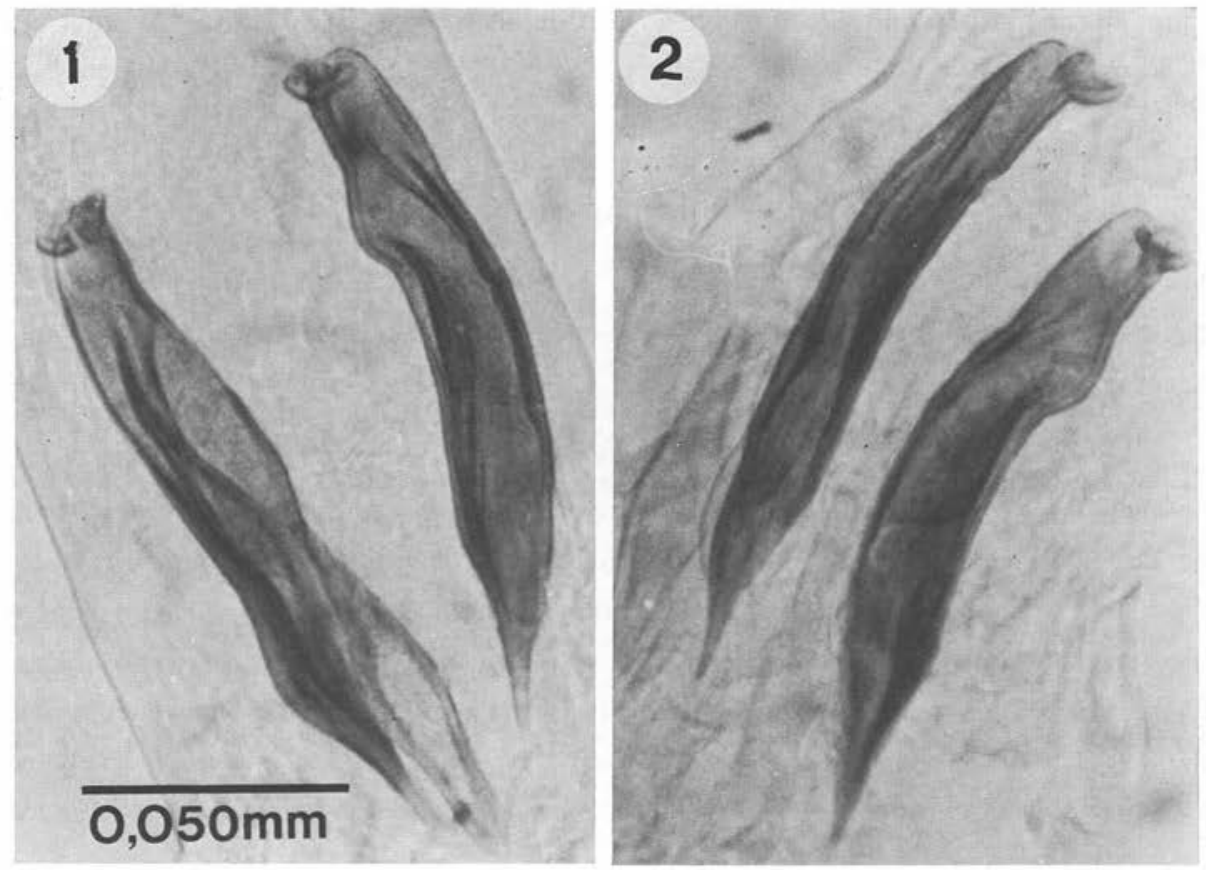

FIG. I. - Trichostrongylus vitrinus : spicules et gubernaculum, vue dorsale $(\times 400)$.

FIG. 2. - Vue latérale du spicule gauche. 


\section{Discussion}

En dehors de Trichostrongylus orientalis qui doit être considéré comme un parasite surtout humain (Biocca, communication personnelle; Ghadirian et Arfaa, 1975), les autres Trichostrongylus infestent habituellement de nombreux animaux (surtout les ruminants), et 7 espèces peuvent être transmises accidentellement à l'homme. Malgré sa distribution gécgraphique étendue chez les bovinés, ovinés, caprinés, camélidés et léporidés, $T$. vitrimus n'a été identifié chez l'homme que dans quelques pays : Chili (Apt et coll., 1967), Iran (Ghadirian et coll., 1968 ; Ghadirian et Arfaa, 1975), U.R.S.S. (Chobanov et coll., 1981) ; et en Afrique, ce nématode n'a jamais été identifié chez l'homme depuis la description princeps de Looss en Égypte (1905).

Les mensurations des 3 vers recueillis chez notre patiente sont plus courtes que celles qui sont indiquées dans la bibliographie et qui concernent des parasites récoltés chez des animaux; ceci peut être dû à leur présence chez un hôte inhabituel. Bien que la femelle recueillie ne puisse être formellement identifiée, la taille des œufs intrautérins permet de supposer fortement qu'il s'agit également de $T$. vitrinus (Cunliffe et Crofton, 1953).

Le bref délai entre le départ de notre patiente du Maroc et son hospitalisation à Lille, élimine la possibilité d'une contamination en France. Nos résultats permettent de présumer qu'au moins 3 espèces de Trichostrongylus peuvent infester l'homme au Maroc.

\section{BIBLIOGRAPHIE}

Apt W., Prudant M., Tagle I., Gonzalez G. : Nuevos casos de trichostrongylosis humana en Chile. Bol. Chil. Parasitol., 1967, 22, 163-165.

Becmeur, LAFFerRe, Lamotte : Les parasitoses intestinales en milieu indigène marocain. Gaz. Med. Fr., 1950, $57,875-880$.

Becouet R., Poirriez J., Dei-Cas E., Dutort E., Deblock S., Abdellatifi M., Vernes A. Contribution à l'étude de la trichostrongylose humaine. (A propos de $7 \mathrm{I}$ observations.) Ann. Soc. Belge Med. Trop., I982, 62, 139-155.

Сhobanov R. E., Safieva L. A., Sadykhov I. A., Kolesnichenko M. L. : New data on trichostrongylids parasitic in man (In Russian). Med. Parazitol., I981, 50, I7-I8.

Clapham P. A. : On the identification of some species of Trichostrongylus. J. Helminthol., 1947, $22,37-46$.

CunlfFFe G., CROFton H. D. : Egg sizes and differential egg counts in relation to sheep nematodes. Parasitology, I953, 43, 275-286.

Ghadirian E., Mofidi C., BiJan H. : Premiers travaux sur l'identification de différentes espèces de Trichostrongylus en Iran. Ann. Parasitol. Hum. Comp., I968, 43, 467-476.

Ghadirian E., ArfaA F. : Present status of trichostrongyliasis in Iran. Am. J. Trop. Med. Hyg., I975, 24, 935-94I.

Looss A. : Notizen zur Helminthologie Aegyptens VI. Das Genus Trichostrongylus n. g., mit zwei neuen gelegentlichen Parasiten des Menschen. Centralbl. Bakteriol., 1905, 39, 409-422.

Nagaty H. F. : The genus Trichostrongylus Looss, 1905. Ann. Trop. Med. Parasitol., 1932, 26, 457-5 18 .

SKRJABIN K. I. : Trichostrongylids of animals and man. Essentials of nematodology. Vol. III. Academy of Sciences of the USSR, Moscow, 1954, 704 p. 